\title{
Dielectrophoretic Cell Concentrator on EWOD-Based Chips
}

\author{
Po-Weng Huang, Tsu-Te Wang, Sheou-Wei Lin, Yu-Chi Chang, and Shih-Kang Fan*, Member, IEEE \\ Institute of Nanotechnology, National Chiao Tung University, Taiwan
}

\begin{abstract}
Dielectrophoretic concentrators were successfully developed on a EWOD-based chip. In such a chip, two major electrokinetic forces were exerted on objects on different scales. EWOD is used to pump droplets on a $\mathrm{mm}$ scale, while DEP manipulates bio-particles on a $\mu \mathrm{m}$ scale. Both cells and polystyrene beads were used to demonstrate the concentration abilities by pDEP and nDEP forces, respectively. Since electrodes are covered with a dielectric layer, instead of creating nonuniform electric fields in the liquid, low frequency signals are consumed in the dielectric layer and providing EWOD. It is necessary to use high frequency signals to generate DEP on a EWOD-based chip. Concentration efficiency, measured by cell count chamber before and after experiments, is around 1.62 with single cycle of pDEP attraction.
\end{abstract}

\section{Keywords-EWOD; DEP; Electrokinetic forces}

\section{INTRODUCTION}

Electrokinetic force is a cross-scale force which is desirable for manipulating object on different scales. EWOD (electrowetting-on-dielectric) and DEP (dielectrophoresis) are two of the examples of electrokinetic manipulations widely used in the lab-on-a-chip applications in recent years. EWOD has been applied in actuation of microdroplets, for its ability to change the surface wettability electrically $[1,2]$. The voltage applied across a dielectric makes an originally hydrophobic (e.g., Teflon) surface to hydrophilic. Creation, transportation, merging, and splitting of droplets were successfully performed on EWOD device, making EWOD a candidate to pump microdroplets of biofluids. However, the hydrophobic interaction may attract and adsorb bio-particles (e.g., cells and proteins) onto the Teflon surface of EWOD devices, resulting in sample reduction and chip contamination.

DEP force, generated from non-uniform electric fields, had been demonstrated in separation, concentration, locating, and orientation bio-objects [3, 4], especially in the research of cellular manipulations. It has been verified that the morphology and life cycle of cells after DEP manipulations were not obviously altered [3, 5]. Non-uniform electric fields are usually generated by electrodes immersed in and directly contacted with bio-fluids. Since dielectric layers are not required in DEP, few studies have been reported on DEP created by electrodes coated with thick dielectrics.

In this research, we would like to take advantages of both the electrokinetic forces, EWOD and DEP, to realize a dielectrophoretic cell concentrator on a EWOD-based chip. In such a device, EWOD actuates droplet on the $\mathrm{mm}$ scale and DEP manipulates the cells on the $\mu \mathrm{m}$ scale. Two major issues need to be considered and resolved: (1) abilities to drive biofluids by EWOD and (2) generation DEP by dielectriccoated electrodes.

\section{PRINCIPLE}

\section{A. $E W O D$}

Surface tension is a dominant force on the microscale. EWOD is a means to change the surface tension between a dielectric solid surface and the liquid above by applying a voltage across the dielectric layer. This phenomenon can be used to transport liquid droplet on a electrode array covered by a dielectric layer and a hydrophobic surface coating. As shown in Fig. 1, a liquid droplet is placed between two glass plates. The space between placed is determined by the thickness of spacers (not shown in Fig. 1). The bottom plate contains an array of driving electrodes, and the top plate has one blank electrode. When applying voltage between the top and bottom plates, the surface on top of the energized driving electrode (second from right) is changed from hydrophobic (the nature of Teflon) to hydrophilic. Therefore, the droplet is moved to the right. The contact angle of the droplet can be expressed by Lippmann and Young equations:

$\cos \theta(V)=\cos \theta_{0}+\frac{\varepsilon_{0} \varepsilon}{2 \gamma_{L G} t} V^{2}$,

where $\varepsilon_{0}$ is the permittivity of vacuum, $\varepsilon$. and $t$ are the permittivity and thickness of the dielectric layer, $\gamma_{L G}$ is the liquid-gas interfacial tension. When a voltage $V$ is applied across the dielectric layer, the contact angle is thereby changed from $\theta_{0}$ to $\theta(V)$. The existence and properties of the dielectric layer are crucial to generate EWOD. A uniform dielectric with no leakage current is necessary.

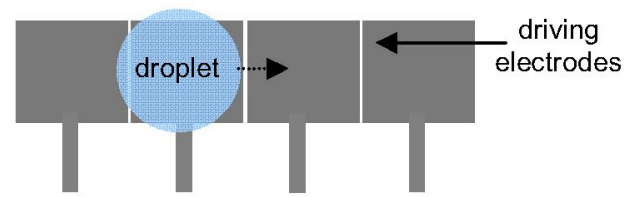

(a) Top view of a EWOD device. Droplet can be pumped by applying voltage on driving electrodes.

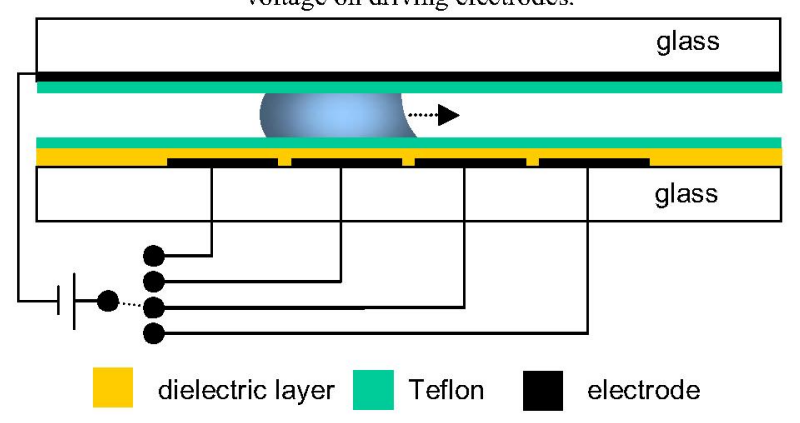

(b) Cross-sectional view of a EWOD device.

Figure 1. Cross-sectional view of a EWOD device: Digital microfluidics manipulated by EWOD. 


\section{B. DEP}

By the advent of microfabrication technique, the manufacturing of microelectrodes are greatly simplified and improved. The well-defined microelectrodes as well as microfluidics technology facilitated the study of DEP. With comparable-sized microelectrodes, DEP can provide sufficient force to investigate objects on the $\mathrm{mm}$ to $\mathrm{nm}$ scale. Hence, DEP was widely applied in biomedical research, clinical diagnosis, and environment analysis recently [6]. The force $\left(F_{D E P}\right)$ exerted on a spherical particle of radius $a$ by DEP can be described by:

$$
F_{D E P}=2 \pi a^{3} \varepsilon_{m} \operatorname{Re}\left(\frac{\mathcal{E}_{p}^{*}-\varepsilon_{m}^{*}}{\varepsilon_{p}^{*}+2 \varepsilon_{m}^{*}}\right) \nabla E^{2},
$$

where $\varepsilon_{p}^{*}$ and $\mathcal{E}_{m}^{*}$ are the complex permittivity of the manipulated particles and the medium, and $E$ is the electric field generated by microelectrodes. The magnitude and direction of DEP forces depend on several factors, including the frequency of the $\mathrm{AC}$ electric field, the conductivity and permittivity of both the particles and the medium where the particles are suspended, and the gradient of the electric field. Depending on the dielectric response of the material to the electric field, an object may be either attracted (positive DEP) toward or repelled (negative DEP) from high field strength region. In an aqueous solution, positive or negative DEP occurs when the dielectric response of the object is higher $\left(\varepsilon_{p}{ }_{p}\right.$ - $\left.\varepsilon_{m}^{*}>0\right)$ or lower $\left(\varepsilon_{p}^{*}-\varepsilon_{m}^{*}<0\right)$ than the solvent (in this case, water), respectively.

\section{DESIGN AND EXPERIMENTS}

\section{A. Concept and Design of Integration}

Although the basic mechanisms of EWOD and DEP are not the same, the similar requirements of microelectrodes inspire us to combine these two electrokinetic forces. The integration of EWOD and DEP is believed to enhance their abilities to explore new research fields. Fig. 2(a) shows the concept to perform both EWOD and DEP with two kinds of electrodes, namely EWOD and DEP electrodes. The stripeshaped electrodes in the center area are mainly used to concentrate cells by DEP. We name stripe-shaped electrodes DEP electrodes. The square-shaped electrodes (shown four in Fig.2(a)) are used to manipulate droplets by EWOD (e.g., transportation, cutting, and merging of droplets). The squareshaped electrodes are called EWOD electrodes. The device cross section is shown in Fig. 2(b), which is similar as that of conventional EWOD devices shown in Fig. 1(b). Both DEP and EWOD electrodes are patterned on the bottom plate and covered with dielectric and hydrophobic layers. The top plate contains a blank electrode with a thin hydrophobic layer deposited on top. DEP electrodes are $100 \mu \mathrm{m}$ in width, and EWOD electrodes are $1 \mathrm{~mm}$ in width. Liquid is $200 \mu \mathrm{m}$ high.

The procedure to concentrate the cells suspended in a liquid droplet is shown in Fig. 1(c)-(e). First, a cell droplet is placed or transported on top of the DEP electrodes by EWOD force. Then voltage is applied on one of the DEP electrodes from left (Fig. 2(c)) to right (Fig. 2(d)) sequentially. The energized DEP electrodes generate non-uniform electric fields (shown by the electric lines), causing cells driven by DEP from left to right. After cells are moved to one side of the droplet (shown right side), the droplet is split into two subdroplets with different cell concentrations by EWOD electrodes (Fig. 2(e)).

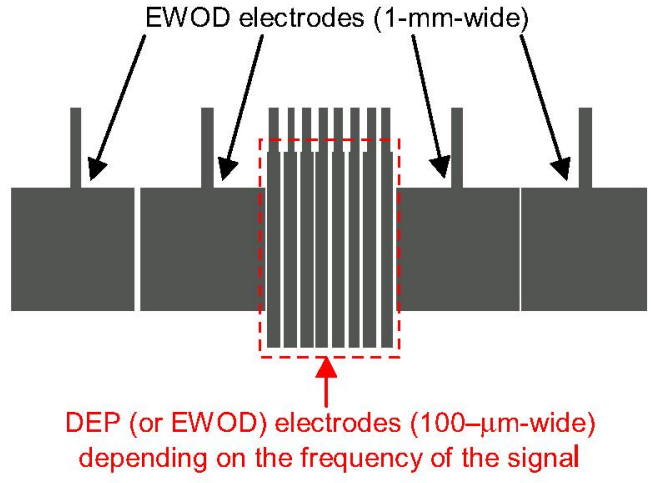

(a) Top view. Striped-shaped electrodes for EWOD or DEP, and squareshaped electrode for EWOD mainly.

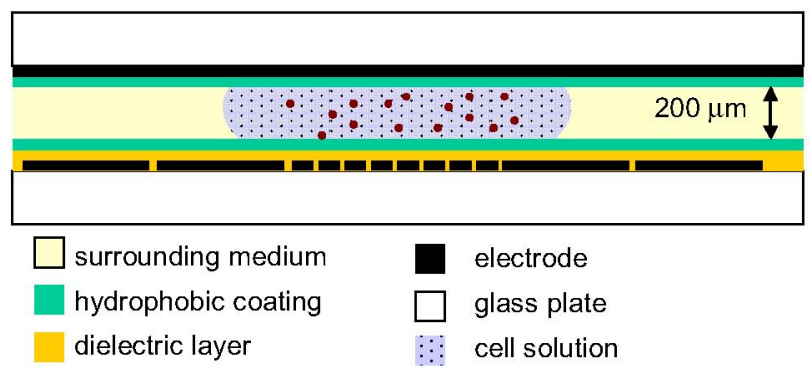

(b) Cross-sectional view.

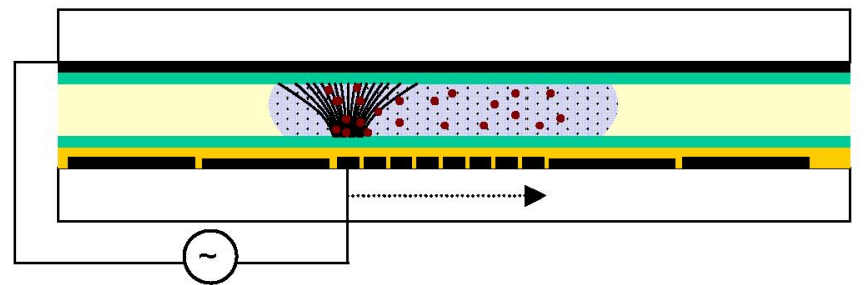

(c) By energizing one of the stripe-shaped electrodes, a non-uniform electric field is created.

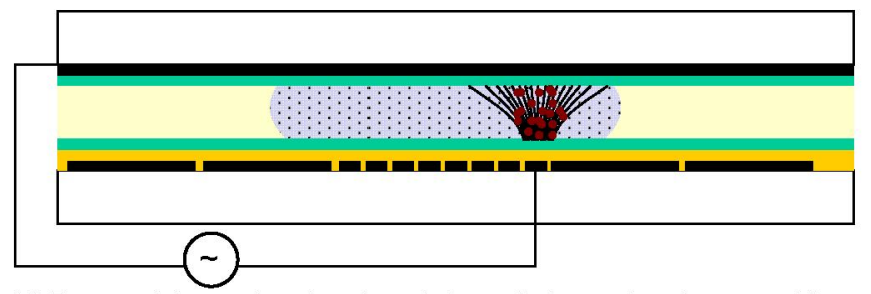

(d) By energizing each stripe-shaped electrode in a series, the non-uniform electric field is transported from left to right.

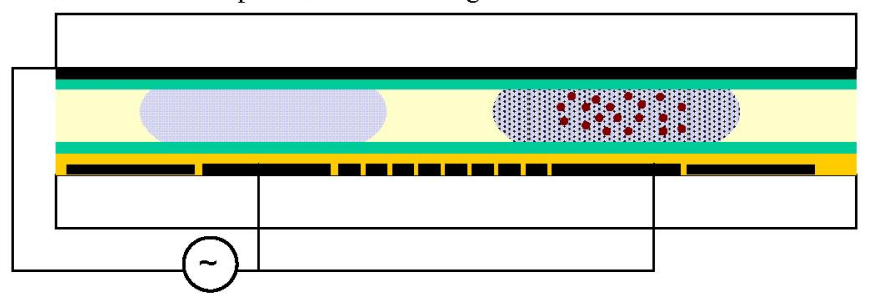

(e) Using EWOD to cut droplet.

Figure 2. The device configuration and procedure to achieve a DEP concentrator on a EWOD device. 


\section{B. EWOD Actuation of Biofluids}

The first attempt to approach our goal is to investigate the ability to pump biofluids by EWOD. Asymmetric EWOD (AEWOD) device [8] was adapted to study pumping of biofluids for its simplicity as shown in Fig. 3(a). The tested fluids include water, PBS (phosphate buffer saline) solution, cells in PBS solution, and $1 \mathrm{mg} / \mathrm{ml} \mathrm{FITC-BSA} \mathrm{(fluorescein}$ isothiocyanate-bovine serum albumin) solution. A drop of the above fluids was dispensed on Teflon and dielectric coated coplanar electrodes (Fig. 3(a)). When applying an AC signal on the electrodes, droplet would be oscillated between the electrodes. By applying same electric signal, we found that the electrolysis is more serious in the PBS solution than in water for PBS has higher conductivity. By using a thicker dielectric with a better quality, electrolysis was eliminated, and PBS solution was successfully pumped by AEWOD. However, pumping of PBS solution containing cells and FITC-BSA solution by AEWOD were not very successful. We observed that after pumping PBS cell solution and FITC-BSA solution for $5 \mathrm{~min}$, the surface became hydrophilic and droplets stopped to oscillate by AEWOD even the voltage was still kept on. By observing the fluorescent images $(15 \mathrm{~min})$, FITCBSA was found adsorbed on the surface above the electrodes. (Fig. 1(b)). The adsorption of bio-particles, or biofouling, occurs more severely on a hydrophobic surface which is necessary for EWOD devices. Preventing biofouling is a fundamental issue to manipulate biofluids by EWOD. A simple way to reduce biofouling adapted in this experiment is to use silicone oil as a surrounding medium. Fig. 3(c) shows the greatly reduced fluorescent signal of FITC-BSA after droplet was spread by AEWOD in silicone oil for $15 \mathrm{~min}$.

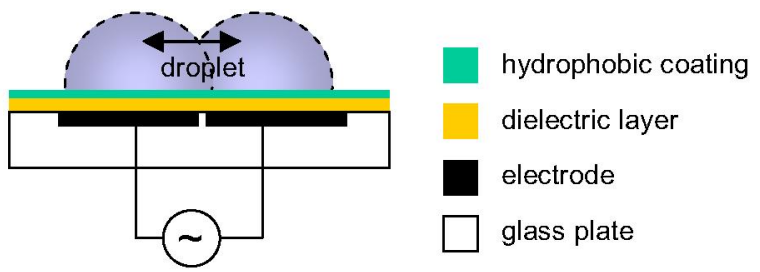

(a) Steup for biofluids EWOD pumping test.

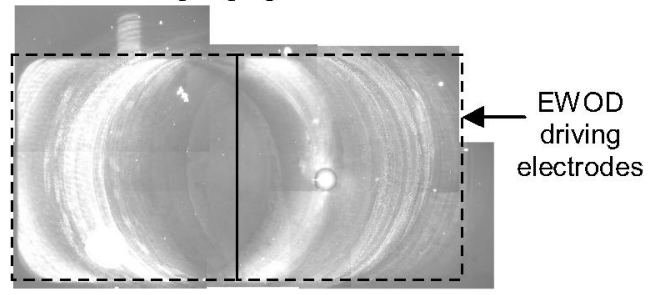

(b) Fluorescent image of a FITC-BSA droplet driven in the air for $15 \mathrm{~min}$ Fluorescent signal represents BSA adsorbed on the hydrophobic surface.

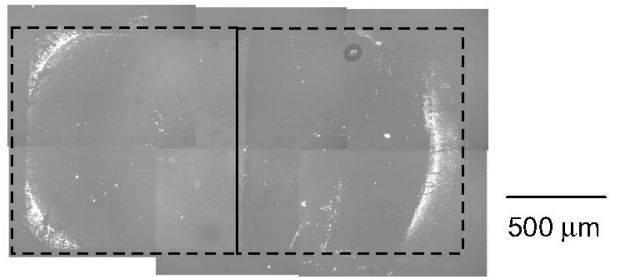

(c) Fluorescent image of a FITC-BSA droplet driven in the oil for $15 \mathrm{~min}$ : less BSA adsorbed on the surface.

Figure 3. Biofluids testing by AEWOD actuation.

\section{DEP Manipulation of Polystyrene Beads and Cells}

The second attempt is to study DEP actuation by dielectric coated electrodes. DEP was investigated by a device shown previously in Fig. 2(a). Polystyrene beads were first tested before using cells. In Fig. 4(a), a liquid containing $5 \mu \mathrm{m}$ polystyrene beads are immersed in an oil medium between two parallel glass plates. The $\mathrm{Au} / \mathrm{Cr}$ electrodes on bottom plate are patterned and covered with a dielectric layer $(5-\mu \mathrm{m}$ thick SU8) and a hydrophobic coating (500-Å-thick Teflon). The top plate has a blank ITO electrode coated with a hydrophobic layer (500-令-thick Teflon). Eight (8) stripeshaped DEP electrodes are in the center of the device, and four (4) square-shaped EWOD electrodes are on the left and right sides.

To manipulate polystyrene beads by DEP, a proper frequency should be applied on one of the DEP electrodes to create a non-uniform electric field. In theory, since the conductivity of beads is much greater than that of water, and the permittivity of the beads is much less than that of water, polystyrene beads should be attracted by positive DEP (pDEP) at low frequency and repelled by negative DEP (nDEP) at high frequency. Experimentally, researchers reported that the pDEP should occur most noticeably at around $1 \mathrm{kHz}$ [7]. However, in our experiment, there was no pDEP observed even applying a DC voltage. A further analysis reveals that at low frequencies, the voltage applied on the DEP electrodes cannot create a non-uniform electric filed in the liquid; most of the voltage is consumed at the dielectric layer to generate EWOD. Therefore, DEP generated by thick dielectric coated electrodes is different from that generated by electrode without or with thin dielectric coating. Only high frequency signals can partially penetrate through the dielectric layer to generate non-uniform electric fields in liquid droplet, causing DEP exerted on suspended objects. As for low frequency signals, applied voltage is mostly depleted in dielectric layer, and EWOD occurs. In the intermediate frequency, both EWOD and DEP can be observed. Although the applicable DEP frequency is restricted DEP still obtained on device with dielectric-coated electrodes. Therefore, EWOD and DEP can be performed on the same device.

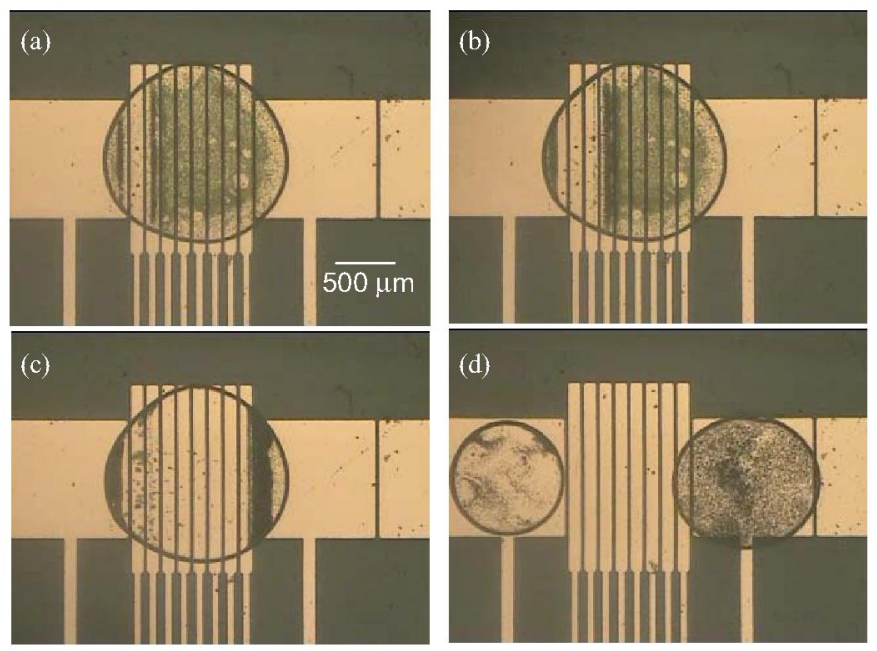

Figure 4. $5 \mu \mathrm{m}$ polystyrene beads were repelled by $\mathrm{nDEP}$ from left to right. The droplet was then cut into two droplets with different bead concentrations. 
Fig. 4(a)-(c) show the manipulation of $5 \mu \mathrm{m}$ polystyrene beads $\left(1.4 \times 10^{8}\right.$ number $\left./ \mathrm{ml}\right)$ by $\mathrm{nDEP}$ forces, with a 42.8 Vrms, $3 \mathrm{MHz}$ AC signal in $50 \mathrm{cSt}$ silicon oil medium. By sequentially applying voltage on one of the DEP electrodes from left to right, the polystyrene beads are repelled from left to right. Finally, most of the polystyrene beads were collected on the right of the droplet (Fig. 4(c)). $84 \mathrm{Vrms}, 1 \mathrm{kHz} \mathrm{AC}$ signal was then applied on the two EWOD electrodes adjacent to DEP electrode to split the original droplet into two subdroplets. As shown in Fig. 4 (d), two droplets with different polystyrene bead concentrations were obtained. It is noteworthy that although the stripe-shaped electrodes are called DEP electrode, they can still be used to pump droplet by EWOD when applying $1 \mathrm{kHz}$ voltage on them. The frequency of the applied signal determines EWOD or DEP to occur.

Concentrating Neuroblastoma cells (Neuro-2a) was also tested on a same device with dielectric-coated EWOD and DEP electrodes. From (2), the conductivity and permittivity of the medium and the particle $\left(\varepsilon_{p}^{*}-\varepsilon_{m}^{*}\right)$ affect the result of DEP. Special care needs to be taken for medium preparation. The prepared medium needs to sustain cells with appropriate conductivity. The medium was prepared by adding $3 \%$ PBS to $280 \mathrm{mM}$ isotonic sucrose solution. The conductivity measured $480 \mu \mathrm{S} / \mathrm{cm}$. The medium was than added to disperse centrifuged Neuro-2a cells for DEP testing. Both pDEP and $\mathrm{nDEP}$ were observed on electrode without dielectric layer coating. For instance nDEP was obtained at $10 \mathrm{kHz}$, while pDEP was achieved at $1 \mathrm{MHz}$. Since the low frequency voltage would be consumed in the dielectric layer, high frequency signals were used to generate pDEP on dielectriccoated electrodes. Fig. 5 shows the video frames of cell concentration.

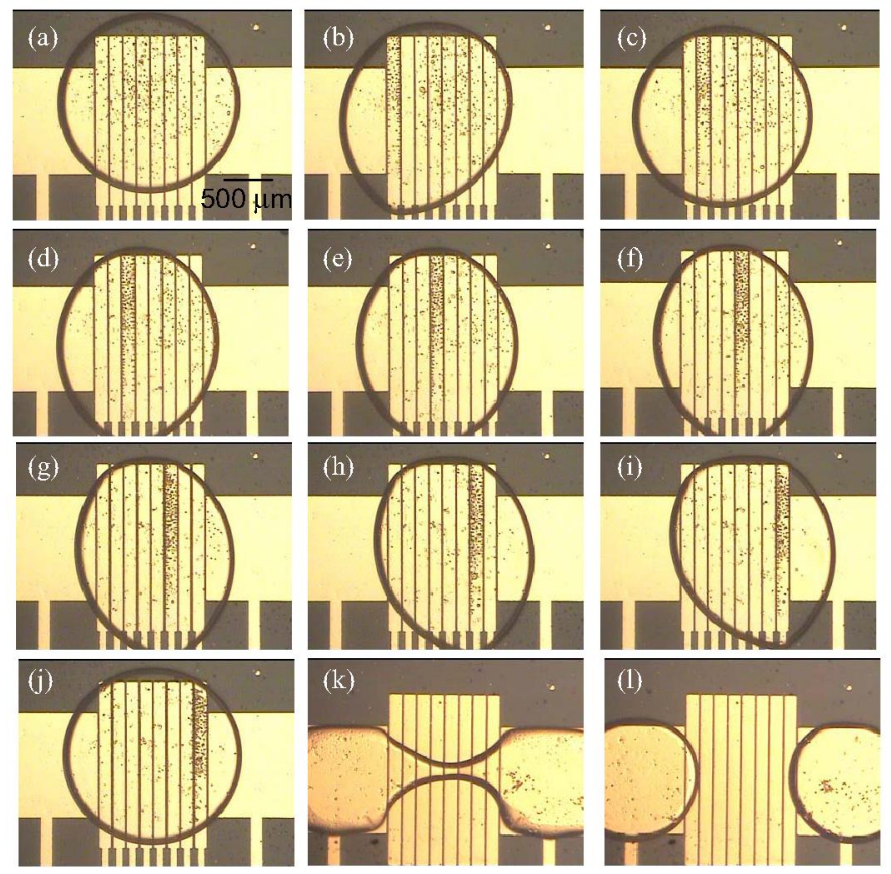

Figure 5 . Nuero-2a cells were manipulated by pDEP in a $480 \mu \mathrm{S} / \mathrm{cm}$ isotonic sucrose medium. (a) cells evenly distributed, (b)-(i) cell concentrated from left to right, (j) the cell concentrated on the right of the droplet, (k)-(l) droplet cut into two droplets with different concentrations.
First, a droplet of Neuro- 2 a cells $\left(8.6 \times 10^{5}\right.$ number $\left./ \mathrm{ml}\right)$ immersed in silicone oil $(50 \mathrm{cSt})$ and placed on the DEP electrodes (Fig. 5(a)). $2 \mathrm{MHz}, 60 \mathrm{Vrms}$ was applied on one of the DEP electrodes from left to right (Fig. 5(b)-(j)). Although pDEP occurs at $1 \mathrm{MHz}, 2 \mathrm{MHz}$ signal was used for $\mathrm{pDEP}$ to eliminate EWOD effect (voltage dropping across dielectric). The droplet was then cut by EWOD ( $84 \mathrm{Vrms}, 1 \mathrm{kHz})$ into two sub-droplets as shown in Fig. 5(k) and (1). By using conventional cell count chamber, we can quantify the cell number of the original droplet and two sub-droplets to calculate the concentration efficiency. The left and the right sub-droplets are in the ratio $1: 4.2$.

\section{CONCLUSIONS}

Cell concentration is a fundamental factor for conventional biological bench assays, such as DNA cloning, plasmid transfection, and cell immunofluorescence staining. Microfluidics process the bench arrays on a miniaturized chip, which saves the reagent, decreases the process time, and making parallel high throughput possible. Electrokinetic forces are suitable for manipulating bioparticles (cells, DNA, proteins) on the $\mu \mathrm{m}$ to $\mathrm{nm}$ scale and microfluidics on the $\mathrm{mm}$ to $\mu \mathrm{m}$ scale. In this paper, we took advantage of the crossscale property of electrokinetic forces and exploited two important electrokinetic forces, EWOD and DEP. EWOD was successfully applied to actuate biofluids droplets, while DEP was used to manipulate the particles or mammalian cells in the droplets. In this manner, the droplets and the bio-particles can be manipulated by electrokinetic forces simultaneously. A cell concentrator was demonstrated to prove the capability of an integrated EWOD-DEP device. However, this cross-scale manipulation ability can be extended to other lab-on-a-chip applications, such as a configurable particle-based bio-assay chip.

\section{REFERENCES}

[1] M. G. Pollack, R. B. Fair and A. D. Shenderov, "Electrowetting-Based Actuation of Liquid Droplets for Microfluidic Applications," Appl. Phys. Lett., vol.77, pp. 1725-1726, 2000.

[2] S. K. Cho, S.-K. Fan, H. Moon, and C.-J Kim, "Toward Digital Microfluidic Circuits: Creating, Transporting, Cutting and Merging Liquid Droplets by Electrowetting-Based Actuation," IEEE Conf. Micro Electro Mechanical Systems (MEMS '02), Las Vegas, NV, 2002, pp. 3235.

[3] H. A. Pohl, Dielectrophoresis, Cambridge University Press, UK, 1987.

[4] L. Zheng, J. P. Brody, P. J. Burke, "Electronic manipulation of DNA, proteins, and nanoparticles for potential circuit assembly," Biosensors and Bioelectronics, vol. 6, pp. 623-641, 2004.

[5] S. Archer, T.T. Li, A. T. Evans, S. T. Britland, and H. Morgan, "Cell reactions to dielectrophoretic manipulation," Biochem. Biophys. Res. Commun., vol. 257, pp. 687-698, 1999.

[6] R. Pethig, "Dielectrophoresis: Using inhomogeneous AC electrical fields to separate and manipulate cells," Critical Reviews in Biotechnology, Vol. 16, no. 4, pp. 331-348. 1996.

[7] J. Auerswald, and H. F. Knapp, "Quantitative Assessment of Dielectrophoresis as a Micro Fluidic Retention and Separation Technique for Beads and Human Blood Erythrocytes," Microelectronic Engineering, vol.67-68, pp. 879-886, 2003

[8] T.-T. Wang, P.-W. Huang, and S.-K. Fan, "Droplets Oscillation and Continuous Pumping by Asymmetric Electrowetting," IEEE Conf. Micro Electro Mechanical Systems (MEMS'06), Istanbul, Turkey, Jan. 2006, pp. 174-177. 